\title{
ORIGINAL
}

\section{BROTE DE TOXIINFECCIÓN ALIMENTARIA POR SALMONELLA ENTÉRICA EN UN ESTABLECIMIENTO DE RESTAURACIÓN COLECTIVA}

Rosa Ma Carbó Malonda (1), Ma Teresa Miralles Espí (1), Rosendo Sanz Bou (1), Federico Mañas Gimeno (1), Silvia Guiral Rodrigo (2) y Elvira Pérez Pérez (1)

(1) Centro de Salud Pública de Alzira. Consellería de Sanitat de la Comunidad Valenciana.

(2) Servicio de Vigilancia y Control epidemiológico. Dirección General de Salud Pública de la Consellería de Sanitat de la Comunidad Valenciana.

\section{RESUMEN}

Fundamento: Se describe un brote de toxiinfección alimentaria por Salmonella enteritidis ocurrido en dos salones de banquetes con ocho grupos de comensales afectados. El objeto de la investigación fue determinar el alimento vehículo de transmisión de la enfermedad.

Métodos: Se analizaron los procesos de elaboración de los alimentos susceptibles de haber causado el brote. La asociación de los alimentos con la enfermedad se analizó mediante un diseño de caso y controles. Se calcularon las Odds Ratio ajustadas (ORa) y sus intervalos de confianza al 95\% (IC95\%) por regresión logística.

Resultados: El número de comensales fue 1.771, distribuidos en 13 grupos durante tres días consecutivos. Se encuestó a 629 personas (36\%). El número de casos probables fue de 250,61 confirmados por salmonella enterica. El biscuit glasé (postre con huevo crudo sin tratamiento térmico) presentó la Odds Ratio ajustada más alta $(\mathrm{ORa}=$ 20,40; IC95\%:7,52-55,30) y fue positivo a Salmonella enterica serotipo enteritidis.

Conclusiones: Existe evidencia epidemiológica y de laboratorio de que el biscuit glacé fue el alimento contaminado que causó el brote. La investigación destacó como factores contribuyentes la utilización de huevo crudo, la producción en grandes cantidades y con antelación al consumo del alimento.

Palabras clave: Brote epidémico. Alimentos, higiene. Intoxicación Alimentaria. Salmonella enteritidis. Restaurantes.
ABSTRACT

\section{Outbreak of Salmonella Enteriditis Food Poisoning at a Dining Hall Facility}

Background: An outbreak of Salmonella enteriditis food poisoning having occurred at two banquet halls, affecting eight groups of guests, is described. This research was aimed at determining the food by means of which this illness was transmitted.

Methods: An analysis was conducted of the food processing procedures subject to having caused the outbreak. The association of the foods with the illness was analyzed by means of a case and control design. The adjusted Odds Ratio (aOR) and the 95\% confidence intervals (CI95\%) were calculated by logic regression.

Results: The guests totalled 1,771 in number, being divided up into 13 groups for three days running. A total of 629 individual $(36 \%)$ were surveyed The probable cases totalled 250 in number, 61 confirmed by Salmonella enterica.The glazed biscuit (dessert with raw egg having undergone no heat processing) showed the highes adjusted Odds Ratio (aOR $=20.40 ;$ CI95\%:7.52-55.30) and tested positive for Salmonella enterica of the enteritidis serotype.

Conclusions: Epidemiological and laboratory evidence supports the glazed biscuit having been the contaminated food having caused the outbreak. The research stressed as contributing factors: the use of raw egg, the production of large quantities ahead of time, prior to the time at which the food item in question was to be eaten.

Key words: Outbreak. Food Poisoning. Salmonella Food Poisoning. Salmonella enteritis. Restaurant industry.

Correspondencia:

Rosa María Carbó Malonda

Sección de situaciones epidémicas y brotes.

Servicio de Vigilancia y control epidemiológico

C/ Micer Mascó, 31-33

46010 Valencia

Correo electrónico: carbo_ros@gva.es 


\section{INTRODUCCIÓN}

Los brotes por Salmonella enteritidis asociados al consumo de alimentos elaborados con huevo crudo siguen siendo frecuentes a pesar de conocerse el riesgo que representa su consumo, de las medidas legales adoptadas y de los programas de educación sanitaria dirigidos a disminuir su incidencia ${ }^{1}$. Esta situación constituye un problema de salud pública importante tanto en España como en otros países europeos y en los Estados Unidos, en cuyos programas de control se incluyen estrategias a adoptar desde los diferentes sectores implicados ${ }^{2,3}$.

En España los brotes estudiados y notificados a la Red Nacional de Vigilancia Epidemiológica entre 1998 y 2001 se relacionan en un $38,5 \%$ con el consumo de huevo y derivados, notificándose un total de 14.815 casos, con 2.106 hospitalizados y cuatro defunciones. De éstos el 85,5\% (1.256 brotes) se asociaron a Salmonella spp y un $52 \%$ (653 brotes) a Salmonella enteritis. Desde 1999 los brotes relacionados con el consumo de huevo y derivados aumentan tanto en el ámbito familiar como el comunitario. La restauración colectiva como lugar de consumo representa entre el 30 y el $35 \%$ de los brotes con ámbito conocido para el periodo 1998$2001^{4}$.

Salmonella enteritis causa en la mayoría de los casos una enfermedad autolimitada pero en personas muy jóvenes, mayores o inmunodeprimidas puede alcanzar la gravedad suficiente como para precisar la hospitalización del paciente, volverse invasiva y causar la muerte ${ }^{1}$. Por otra parte su presentación en forma de brotes comunitarios en restauración colectiva a gran escala puede suponer un problema relevante tanto por la carga de enfermedad como por la trascendencia socioeconómica para el sistema sanitario y los afectados ${ }^{4}$. La persistencia de estas toxiinfecciones de ámbito colectivo y la implicación de alimentos que utilizan huevo crudo en su elaboración aconsejan pro- fundizar en la investigación y prevención de estos brotes.

El objetivo del estudio es presentar la investigación epidemiológica y de laboratorio de un brote de toxiinfección alimentaria que permitió la confirmación de la hipótesis del postre biscuit glacé, elaborado con huevo crudo, como el alimento vehículo de la infección, así como valorar las medidas adoptadas para su control con el fin de evitar su recurrencia.

\section{SUJETOS Y MÉTODOS}

El día 14 de mayo de 2002 se notificó la existencia de un brote de toxiinfección alimentaria con 32 casos entre los asistentes a diferentes banquetes celebrados en dos salones de diferentes localidades pertenecientes al mismo propietario y ubicadas en el área de salud de Alzira en la Comunidad Valenciana. La investigación se inició de manera inmediata con el fin de describir la situación, identificar el alimento implicado, conocer el agente causal y adoptar las medidas de control pertinentes.

La investigación se planteó con un enfoque multidisciplinar: epidemiológico, ambiental y microbiológico. Tras la visita de inspección a los locales por parte de los técnicos del Centro de Salud Pública, epidemiólogo y veterinario, se identificó un total de 12 banquetes celebrados los días 10, 11 y 12 de mayo, y un grupo de manipuladores, estimándose un total de 1.771 comensales y detectándose afectados en nueve de estos grupos. Los menús consumidos y los manipuladores de alimentos coincidían en gran parte por lo que se decidió realizar un estudio conjunto del brote.

\section{Investigación epidemiológica}

Se realizaron las encuestas epidemiológicas para el estudio de brotes de enfermeda- 
des transmitidas por alimentos, utilizando el cuestionario contenido en el protocolo de la Red Nacional de Vigilancia Epidemiológi$\mathrm{ca}^{5}$ con las variables clínico-epidemiológicas y de laboratorio, añadiendo todos los alimentos consumidos en cada uno de los banquetes. Se propuso una cobertura de encuestas del $50 \%$, entre enfermos y controles proporcional a la distribución de los asistentes en los diferentes banquetes. En aquellos banquetes en los que no se declararon casos se realizó un muestreo a conveniencia de un $5 \%$ de los asistentes. Las encuestas fueron realizadas telefónicamente por siete técnicos de salud pública.

Definición clínica de caso: se definió como caso probable al enfermo que presentaba al menos dos de los siguientes síntomas: tres o más deposiciones líquidas al día, dolor abdominal, fiebre alta, náuseas, y/o vómitos, que podían estar acompañados por dolor generalizado y cefalea y que había asistido a alguno de los banquetes celebrados en los salones implicados durante el periodo de estudio. La definición de caso confirmado correspondía al caso probable con coprocultivo positivo a Salmonella spp.

Los controles se seleccionaron entre los comensales a partir de los listados facilitados por los contratantes de los banquetes, con el criterio de haber consumido alguno de los alimentos de los menús y no haber padecido la enfermedad.

La investigación se planteó en una primera fase como un estudio descriptivo de la forma de presentación de la enfermedad en cuanto a características clínicas y epidemiológicas. Se determinaron las características personales de los enfermos, la sintomatología presentada, la distribución temporal mediante la curva epidémica en función de la hora y fecha de inicio de los síntomas, la media y rango del período de incubación. Se estudió la distribución de los enfermos en cada banquete y fecha de exposición con relación al número de comensales estimados en cada uno de ellos, calculando la tasa de ataque por banquete o grupo de exposición (núm de enfermos/núm de comensales). La asociación de la enfermedad con cada alimento se estudió mediante un diseño de casos y controles, con una relación casocontrol como mínimo de 1:1 y se determinaron las Odds ratio (OR) con intervalos de confianza del 95\%. Para estudiar la contribución independiente de cada alimento se calcularon las OR. ajustadas (ORa) por el resto de alimentos implicados, edad y sexo mediante un modelo de regresión logística no condicional. El análisis estadístico se realizó con el programa SPSS v.10.

Se valoró la existencia de relación causal mediante la aplicación de los criterios de causalidad de Bradford Hill ${ }^{6}$ para reforzar la validez externa de los resultados de la investigación.

\section{Investigación ambiental}

Se llevó a cabo una inspección de los locales y cocinas para conocer las condiciones higiénico-sanitarias, estructurales y de procedimiento. Se investigó la procedencia y secuencia de preparación de todos los alimentos, su conservación y medio de transporte. La preparación de los alimentos se evaluó según los estándares europeos de higiene alimentaria (HACCP: Hazard Analysis and Critical Control Point) ${ }^{7}$. Los alimentos que por su procedimiento de elaboración eran considerados de mayor riesgo fueron analizados mediante la elaboración de diagramas de flujo y evaluación y definición del riesgo.

Teniendo en cuenta que en fechas muy cercanas se esperaba celebrar nuevos banquetes en estos salones se aplicaron medidas cautelares inmediatas. Se realizó una encuesta epidemiológica a todos los manipuladores para conocer la actividad desarrollada con respecto a los menús elaborados y/o servidos, antecedentes recientes de 
enfermedad susceptible de transmisión por alimentos y consumo de alimentos de los menús. Los manipuladores con sospecha de enfermedad fueron excluidos de la manipulación de alimentos.

\section{Investigación microbiológica}

Se recuperaron 23 resultados de coprocultivos positivos a Salmonella procedentes de los diferentes hospitales que habían atendido a los enfermos y se recogieron 52 muestras de enfermos para coprocultivo.

Se efectuó coprocultivo a 33 manipuladores (30 sanos y tres enfermos) con recogida de tres muestras de heces correspondientes a tres días consecutivos.

Se recogieron muestras testigo de los postres biscuit glacé y tarta que correspondían a los servidos en los banquetes a estudio. El biscuit glacé había sido elaborado en las cocinas de los salones y la tarta procedía de una pastelería externa. También se recogieron muestras de otros alimentos en origen, que entre ellos incluía huevos procedentes de diferente lote a los utilizados en la elaboración del biscuit glacé por no quedar restos de los anteriormente utilizados para la elaboración de este postre.

Los análisis de alimentos, manipuladores y enfermos se realizaron en el laboratorio de Salud Pública de Valencia siguiendo métodos estándar para cultivo y serotipado.

\section{RESULTADOS}

El número de comensales en estudio fue de 1.771, distribuidos en 13 grupos, 12 banquetes y un grupo de manipuladores, con tres fechas de exposición (días 10, 11 y 12 de mayo) y dos salones de los mismos propietarios en dos localidades cercanas. Los enfermos residían en 25 municipios diferentes.
El número total de encuestados fue de 629 personas, lo que supone un $36 \%$ de los comensales estimados. El número de enfermos, casos probables, fue de 250 siendo 61 de ellos confirmados por laboratorio para Salmonella. Un total de 119 personas fueron atendidas en los servicios de urgencias de los centros de salud y hospitales. Fueron hospitalizados 12 enfermos.

La edad media de los enfermos fue de 40 años $(\mathrm{DE}=20)$, con un rango entre 2 y 82 años, frente a una edad media de los controles de 38 años $(\mathrm{DE}=20)$, con un rango entre 2 y 78 años. La distribución por género entre los enfermos fue de un 41\% (IC95\%: $35 \%$ $48 \%$ ) de hombres frente a un 59\% (IC95\%: $52 \%-65 \%$ ) de mujeres y entre los controles de un 51\% (IC95\%: 46\%-57\%) de hombres frente a un $49 \%$ (IC95\%: 43\%-54\%) de mujeres. El análisis bivariado de la asociación de la edad y el sexo con la presencia de enfermedad no resultó estadísticamente significativo.

El periodo de incubación presentó un tiempo medio de 28 horas, con un valor mínimo de 9 horas y un máximo de 78 horas. La sintomatología referida por los pacientes fue: diarrea (94\%), dolor abdominal $(91 \%)$, fiebre $(76 \%)$, náuseas $(41 \%)$, vómitos (34\%) y otros como cefalea y malestar.

El primer caso inició síntomas a las 12 horas del día 11 de mayo y el último caso a las 16 horas del día 15 del mismo mes. La distribución temporal se estudió agrupando los casos según fecha de inicio de síntomas en intervalos de 12 horas. En la figura 1 se representan dos curvas epidémicas, una por cada salón de banquetes.

Se estudió a los encuestados con relación a todos los alimentos elaborados y/o consumidos en alguno de los salones y días de exposición, con un total de 54 alimentos analizados. De los 12 banquetes estudiados se declararon casos en ocho y en éstos últimos estaba presente el postre biscuit glacé 
Figura 1

Curva epidémica para cada salón de banquetes

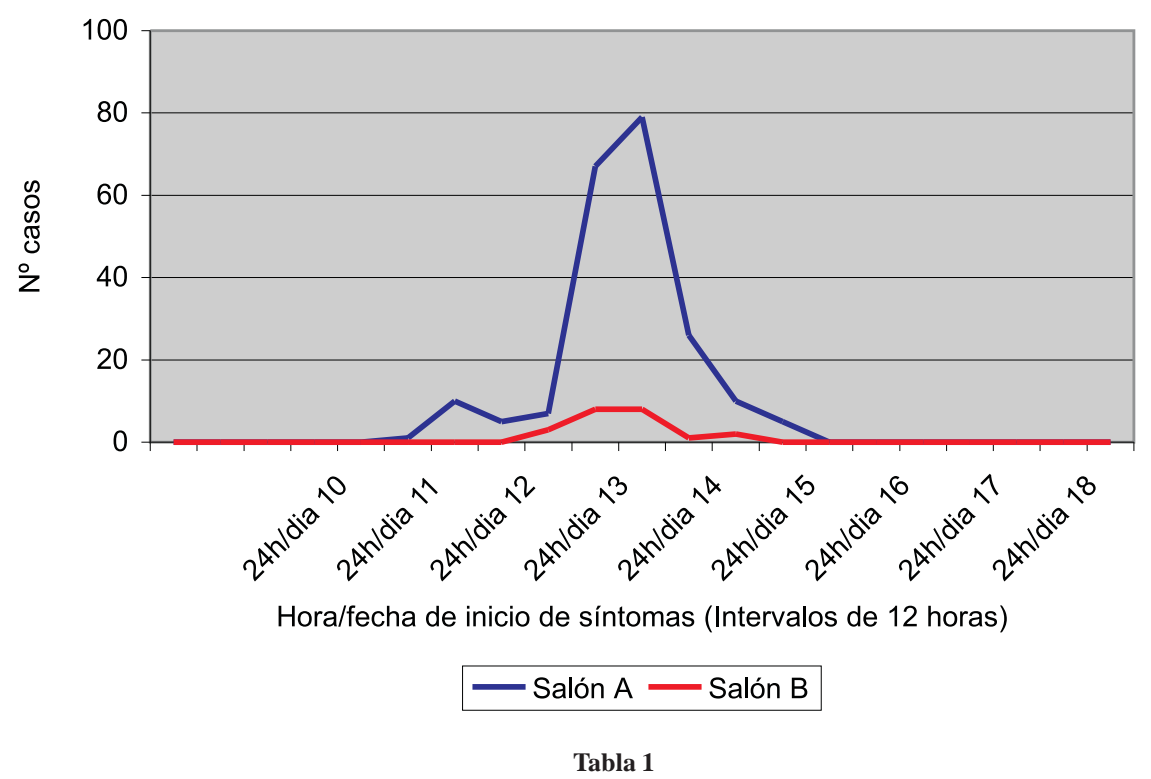

Tasas de ataque por fechas, grupos de exposición y casos confirmados

\begin{tabular}{|c|c|c|c|}
\hline Fecha de exposición & $\begin{array}{c}\mathbf{N}^{\mathbf{0}} \text { expuestos } \\
\text { por banquete }\end{array}$ & $\begin{array}{c}\text { Tasas de ataque } \\
\text { en \%* }\end{array}$ & $\begin{array}{c}\mathbf{N}^{\mathbf{0}} \text { casos confirmados por } \\
\text { laboratorio a Salmonella }\end{array}$ \\
\hline $10-05-02$ & 200 & 9,5 & 3 \\
\hline $11-05-02$ & 300 & 0,3 & - \\
\hline $12-05-02$ & 100 & - & 9 \\
\hline $12-050-2$ & 116 & 41,4 & 18 \\
\hline $12-05-02$ & 229 & 26,2 & 7 \\
\hline $12-05-02$ & 57 & 42,1 & 6 \\
\hline $12-05-02$ & 82 & 37,8 & 2 \\
\hline $12-05-02$ & 128 & 31,3 & 2 \\
\hline $12-05-02$ & 44 & 20,5 & - \\
\hline $12-05-02$ & 166 & 8,4 & - \\
\hline $12-05-02$ & 138 & - & 60 \\
\hline $12-05-02$ & 173 & - & \\
\hline & Total & & \\
\hline
\end{tabular}

* Tasa de ataque: $\mathrm{n}^{\mathrm{o}}$ enfermos / $\mathrm{n}^{\mathbf{0}}$ comensales. No incluye manipuladores.

formando parte del menú. En los tres banquetes restantes donde no se habían producido casos no constaba el biscuit glacé en el menú. Las tasas de ataque entre los comensales de los banquetes celebrados los días 10 y 11 fueron menores que las tasas de los banquetes celebrados durante el día 12 del periodo a estudio (tabla 1). Los alimentos que presentaron valores de la odds ratio estadísticamente significativos en el análisis 
Tabla 2

Diferencia de tasas de ataque entre los enfermos que consumieron y no consumieron, y análisis de la asociación consumo-enfermedad con resultado estadísticamente significativo

\begin{tabular}{|l|c|c|c|}
\hline Alimento & $\begin{array}{l}\text { Diferencia de } \\
\text { tasas de ataque }\end{array}$ & $\begin{array}{c}\text { Odds Ratio } \\
\text { cruda }\end{array}$ & $\begin{array}{c}\text { O.R. cruda } \\
\text { IC 95\% }\end{array}$ \\
\hline Lechuga & 20,36 & 2,28 & $2,26-27,49$ \\
\hline Jamón & 16,49 & 2,08 & $1,32-3,27$ \\
\hline Queso manchego & 17,98 & 2,13 & $1,39-3,26$ \\
\hline Cigalas & 24,63 & 2,97 & $2,05-4,31$ \\
\hline Rojos & 21,46 & 2,64 & $1,74-4,02$ \\
\hline Langostinos & 11,45 & 1,63 & $1,14-2,32$ \\
\hline Bacalao milanesa & 19,27 & 2,19 & $1,21-3,98$ \\
\hline Ternera & 9,35 & 1,48 & $1,05-2,11$ \\
\hline Melocotón & 22,00 & 2,45 & $1,13-5,36$ \\
\hline Biscuit glacé & 44,35 & 22,31 & $9,04-70,94$ \\
\hline Tarta A & 12,84 & 1,72 & $1,22-2,43$ \\
\hline Tarta B & 22,59 & 2,51 & $1,49-4,23$ \\
\hline Tarta C & 23,07 & 2,56 & $1,19-5,57$ \\
\hline Vino tinto & $-13,01$ & 0,58 & $0,42-0,84$ \\
\hline Licores & $-16,82$ & 0,47 & $0,22-0,98$ \\
\hline Menú de niños & $-22,73$ & 0,33 & $0,18-0,62$ \\
\hline
\end{tabular}

bivariante fueron 13, siendo el biscuit glacé el que presentó mayor diferencia en las tasas de ataque y también el valor más alto de la O.R cruda $(\mathrm{OR}=22,31)$, seguido de las cigalas $(\mathrm{OR}=2,97)$ (tabla 2). El ajuste mediante regresión logística por los alimentos que habían resultado significativos en el análisis bivariado presentó al biscuit glacé y a las cigalas como factores de riesgo y al consumo de vino tinto como factor protector, independientemente de la edad, sexo y del consumo del resto de alimentos (tabla 3 ).
Las muestras de heces de los enfermos fueron procesadas en ocho laboratorios clínicos de los diferentes hospitales y centros de referencia. Las muestras analizadas corresponden a 75 comensales enfermos con 60 resultados positivos a Salmonella. En cuanto a los manipuladores se estudiaron muestras de heces de 33 manipuladores, tres enfermos y 30 sanos. Fueron positivos a Samonella entérica subespecie I serotipo Enteritidis (9,12:g,m:-) un total de cinco manipuladores, siendo cuatro de ellos sanos

Tabla 3

Análisis multivariante del consumo-enfermedad con resultado significativo

\begin{tabular}{|l|c|c|}
\hline Alimento & $\mathbf{p}$ & OR ajustada (IC 95\%) \\
\hline Biscuit glacé & $<0,05$ & $20,40(7,52-55,30)$ \\
\hline Cigalas & $<0,05$ & $2,94(1,80-4,80)$ \\
\hline Vino tinto & $<0,05$ & $0,40(0,27-0,61)$ \\
\hline
\end{tabular}


Tabla 4

Resultados de la investigación de muestras de heces y alimentos por laboratorio

\begin{tabular}{|c|c|c|c|}
\hline Procedencia & $\mathrm{N}^{0}$ muestras & Positivas & Tipo de Salmonella \\
\hline Comensales Enfermos & 75 & 60 & $\begin{array}{l}\text { 37 Salmonella Enterica serotipo Enteritidis }(9,12: \mathrm{g}, \mathrm{m} .-) \\
21 \text { Salmonella spp } \\
2 \text { Salmonella D }\end{array}$ \\
\hline Manipuladores & $\begin{array}{c}3 \\
30\end{array}$ & $\begin{array}{l}1 \\
4\end{array}$ & $\begin{array}{l}\text { 1 Salmonella Enterica serotipo Enteritidis }(9,12: \mathrm{g}, \mathrm{m}-) \\
4 \text { Salmonella Enterica serotipo Enteritidis }(9,12: \mathrm{gm}-)\end{array}$ \\
\hline $\begin{array}{l}\text { Alimentos } \\
\text { Biscuitglacé } \\
\text { Tarta }\end{array}$ & $\begin{array}{l}2 \\
2\end{array}$ & 2 & 2 Salmonella Enterica serotipo Enteritidis (9,12:gm-) \\
\hline Alimentos en origen & 7 & - & - \\
\hline
\end{tabular}

(4/30) y uno enfermo (1/3). Dado que los resultados procedían de diferentes laboratorios se informaron las siguientes subespecies y tipos: Salmonella entérica subespecie I serotipo: Enteritidis $(9,12: \mathrm{g}, \mathrm{m}:-)$ en 42 muestras; Salmonella spp en 21 muestras y Salmonella $D$ en dos muestras (tabla 4).

Los manipuladores que presentaron algún síntoma de enfermedad gastrointestinal fue- ron excluidos de la manipulación, en aplicación del Real Decreto 202/2000, de 11 de febrero, por el que se establecen las normas relativas a los manipuladores de alimentos. A los trabajadores sanos que resultaron con coprocultivo positivo a Salmonella se les indicó el cese en la actividad y fueron remitidos a su médico de cabecera para el tratamiento antibiótico correspondiente como portadores sanos de Salmonella. Después de

Figura 2

Esquema de preparación del Biscuit glacé

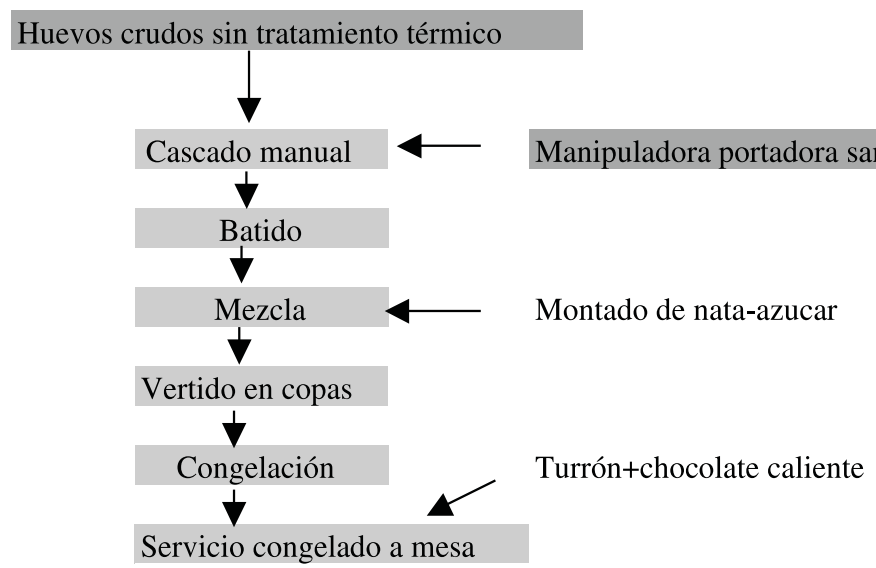

Elementos potenciales de contaminación

PCC: Operación o intervalo con peligro de contaminación o crecimiento de Salmonella.

Desde cascado a congelación se exige: menor tiempo posible y temperaturas de refrigeración. 
Tabla 5

Aplicación de los criterios de causalidad de Bradford Hill a la hipotética relación causal entre el consumo de biscuit glacé y la ocurrencia del brote

\begin{tabular}{|l|l|}
\hline Criterio & Evidencia \\
\hline $\begin{array}{l}\text { Fuerza de la } \\
\text { asociación }\end{array}$ & $\begin{array}{l}\text { Odds Ratio ajustadas con valor muy alto y estadísticamente significativo para el biscuit glacé } \\
\text { (OR a }=20,396 ; \text { IC95\%:7,523-55,297). }\end{array}$ \\
\hline Consistencia & $\begin{array}{l}\text { Otros brotes con estudio de la asociación entre el consumo de alimento con huevo crudo y } \\
\text { salmonelosis presentan resultados de las medidas de asociación similares a la presente } \\
\text { investigación }\end{array}$ \\
\hline Especificidad & $\begin{array}{l}\text { De las } 256 \text { personas que consumieron biscuit glacé 240 resultaron enfermas por } \\
\text { Salmonelosis }\end{array}$ \\
\hline Temporalidad & $\begin{array}{l}\text { La curva epidémica bimodal presenta forma típica de exposición a fuente común para cada } \\
\text { día de exposición, teniendo en cuenta el tiempo medio de incubación de Salmonelosis }{ }^{17}\end{array}$ \\
\hline Gradiente biológico & $\begin{array}{l}\text { Las tasas de ataque fueron mayores en los banquetes celebrados el día 12 frente a las tasas de } \\
\text { ataque entre los comensales de los días 10 y 11. }\end{array}$ \\
\hline $\begin{array}{l}\text { Plausibilidad y } \\
\text { coherencia }\end{array}$ & $\begin{array}{l}\text { Es bien conocida la transmisión de la Salmonella a partir del consumo de alimentos con } \\
\text { huevo crudo sin tratamiento térmico posterior y su mantenimiento a pesar de la conservación } \\
\text { del alimento a baja temperatura }\end{array}$ \\
\hline Experimentación & $\begin{array}{l}\text { A partir de la adopción de las medidas de intervención cautelares por parte de la inspección } \\
\text { veterinaria no se produjeron nuevos casos de Salmonelosis en los sucesivos e inmediatos } \\
\text { banquetes. }\end{array}$ \\
\hline Analogía & $\begin{array}{l}\text { Están publicados brotes de toxiinfección alimentaria que encuentran asociación entre otros } \\
\text { alimentos, diferentes al biscuit glacé, elaborados también con huevo crudo }{ }^{16} \text {. }\end{array}$ \\
\hline
\end{tabular}

quince días de haber terminado el tratamiento se les practicó un nuevo coprocultivo de control y tras la negativización de los resultados se les emitió el alta epidemiológica.

La investigación ambiental descubrió puntos críticos en la elaboración de varios alimentos pero, en cambio, éstos habían sido elaborados con tratamiento térmico previo e inmediato a su servicio a los comensales. En el caso del biscuit glacé se había utilizado huevo crudo para su elaboración, sin tratamiento térmico y posteriormente se mantenía congelado. Se constató la deficiencia en la limpieza y desinfección en el desmontaje de las máquinas montadoras del biscuit glacé. Además, en su elaboración directa participó una manipuladora sana que resultó con coprocultivo positivo a Salmonella. El procedimiento de elaboración de este alimento se presenta en la figura 2 .

El laboratorio del Centro de Salud Pública de Valencia realizó las determinaciones de los alimentos con los siguientes resultados: dos muestras de biscuit glacé, una de cada salón, positivas a Salmonella entérica subespecie I serotipo: Enteritidis $(9,12$ :g,m.- $)$, dos muestras de tarta negativas a los parámetros habituales y siete muestras de alimentos en origen, que incluía los huevos de diferente lote a los utilizados, también negativas (tabla 4).

La aplicación de los criterios de causalidad de Bradford Hill a los resultados de la investigación ayudó a valorar la hipotética relación causal entre el consumo de biscuit glacé y la ocurrencia del brote (tabla 5).

\section{DISCUSIÓN}

Existen evidencias epidemiológicas, estadísticas, microbiológicas y de causación que apoyan la relación causal entre el consumo de biscuit glacé y la ocurrencia del brote.

La utilización para el análisis de la definición clínica de caso es posible que haya introducido algunos errores de clasificación, ya que ésta aumenta la sensibilidad frente a la especificidad, pero el elevado número de coprocultivos positivos a Salmonella hace 
pensar que no afectó a la validez interna del estudio. Tampoco se consideran importantes los errores de clasificación de la exposición ya que la recogida de datos se realizó en un tiempo relativamente corto tras la detección del brote. Por otra parte los controles fueron elegidos del entorno de los casos lo que evitaría los sesgos de selección.

La investigación del agente y vehículo de transmisión no presentó dificultades para su identificación, ya que desde las primeras fases de la investigación se conocía la inexistencia de casos en otros banquetes, en los mismos salones, en los que no se había servido biscuit glacé. Por otra parte, los niños enfermos habían consumido un menú diferente a los adultos excepto el postre que habían compartido con sus familiares adultos. Estas observaciones fueron las que condujeron a plantear desde el inicio la hipótesis del biscuit glacé como el principal alimento sospechoso. El conocimiento de su proceso de elaboración y la indeterminación de las condiciones de mantenimiento reforzaron esta sospecha. En su cadena de producción podían existir varios puntos críticos, desde el cascado manual de los huevos hasta su congelación y posterior presentación a la mesa. Por otra parte no había evidencias concretas del lugar de elaboración ni si hubo traslado entre las cocinas de los diferentes salones. Tampoco se pudo definir los tiempos transcurridos desde la elaboración hasta el consumo. Sin embargo, esta circunstancia junto a la elaboración en grandes cantidades, sin revisión de las condiciones higiénicas del aparataje utilizado, hizo sospechar que se elaboró con gran antelación a su consumo, lo que pudo contribuir al crecimiento de la Salmonella previamente a la congelación ${ }^{8,9,10}$. Este hecho podría explicar las diferentes tasas de ataque según los días de exposición. La existencia de muestra testigo de biscuit, correspondiente a la producción servida en los menús a estudio y conservada por el responsable del establecimiento, permitió confirmar la hipótesis de este alimento como vehículo de transmisión. Con respecto a los factores que hicieron posible la contaminación del biscuit glacé no se descarta que fuera en origen ${ }^{9,11}$, pero no se pudo confirmar por no disponer de muestras de huevos procedentes del mismo lote que los utilizados. En contra de la hipótesis del huevo como fuente de Salmonella para el biscuit glacé tendríamos la participación directa de una manipuladora portadora sana de Salmonella en su elaboración, pero tampoco permite afirmar su contribución en la transmisión por desconocer su estado de portadora previo y formaba parte del grupo de expuestos. Además su contribución en la contaminación es menos plausible ya que las deficiencias higiénicas deberían haber sido importantes para que tuviera lugar la transmisión. Por otra parte los manipuladores enfermos iniciaron síntomas tras el consumo de los alimentos de los menús servidos en el periodo a estudio.

El Ministerio de Sanidad y Consumo en España publicó en 1991 en el Real Decreto 1.254 unas pautas de preparación y conservación de alimentos que lleven huevo como ingrediente y la obligatoriedad de su sustitución por ovoproductos pasteurizados ${ }^{12}$. El Real Decreto 202/2000, sobre normas relativas a los manipuladores de alimentos ${ }^{13}$, dispone, como estrategia de prevención, la formación continuada en las prácticas correctas, que debe quedar incluida en el Plan de análisis de peligros y puntos de control crítico de la empresa del sector alimentario, definiendo el ámbito de los manipuladores de alto riesgo, así como el control y la supervisión por la autoridad competente. Actualmente la Agencia Española de Seguridad Alimentaria ha adoptado la promoción de la seguridad alimentaria como un aspecto fundamental de la salud pública, ofreciendo garantías e información objetiva a los consumidores y a los agentes económicos del sector agroalimentario y la fomenta y dirige con el fin de asegurar la inocuidad de los alimentos desde el principio hasta el final de la cadena alimentaria ${ }^{14}$.

Con el fin de prevenir la aparición de nuevos casos de enfermedad, teniendo en cuen- 
ta que en fechas muy cercanas se esperaba la celebración de nuevos banquetes en los mencionados salones, se procedió a la vigilancia exhaustiva del cumplimiento estricto de todas las condiciones concretadas en el Real Decreto 202/200013 el cual fue implementado con las siguientes medidas:

- Autorización previa por parte de las autoridades sanitarias de los menús a elaborar que en ningún caso debían llevar salsas ni cremas. No se autorizó ningún menú que incluyera platos de riesgo potencial, expresamente el biscuit glacé.

- Desinfección previa, con solución clorada, de todas las superficies que entraban en contacto con las manos y no son desinfectadas habitualmente: pomos y manillas de puertas, interruptores de luz, mandos y utensilios de cocina.

- Preparación y cocinado de los platos por personal debidamente equipado de guantes y mascarilla.

- Todos los manipuladores que no pudieron acreditar formación mínima recibieron un curso de formación específico sobre «Manipulación de alimentos del sector de comidas preparadas» por una empresa acreditada.

- Asignación de dos inspectores veterinarios para la vigilancia exhaustiva desde el día 16 hasta el día 19 domingo, de las medidas preventivas en los procesos de preparación de los platos y de la correspondencia de estos con los menús programados.

La aplicación de las medidas excepcionales, basadas en la gestión del riesgo por el principio de precaución ${ }^{15}$, en las fases iniciales de la investigación, cuando sólo se disponía de una hipótesis de sospecha del vehículo y de sus factores contribuyentes, fue probablemente el factor determinante para evi- tar la ocurrencia del brote en los sucesivos banquetes.

Los resultados de laboratorio de los diferentes coprocultivos no son específicos pero los ocho hospitales que informaron resultados incluyeron la Salmonella aislada en el grupo de Salmonella entérica.

El efecto protector del consumo de vino tinto, analizada como variable dicotómica consumo si/no, es uno de los hallazgos de nuestro estudio consistente con los resultados de otras investigaciones de brotes causados por Salmonella ${ }^{18}$.

La investigación epidemiológica y de laboratorio permitió determinar al biscuit glacé como el alimento vehículo de la transmisión. La investigación ambiental permitió identificar los puntos críticos de la elaboración de los alimentos implicados. El origen de la contaminación no pudo ser determinado. La exhaustividad de las medidas cautelares evitó probablemente la recurrencia del brote. A pesar de las reglamentaciones y recomendaciones vigentes se considera prioritario para la prevención incrementar el esfuerzo entre todos los sectores implicados, desde el control de las fuentes de la Salmonella en los alimentos en origen, hasta la observancia estricta de las reglamentaciones en la manipulación de alimentos mediante el control por los servicios de inspección sanitaria.

\section{BIBLIOGRAFIA}

1. Gein T, O'Flanagan D, McCarthy T, Prendesergast T. Reducción del riesgo de salmonelosis procedente de los huevos. Eurosurveillance 1997; 2:86-88.

2. Salmonella enteritidis en Europa occidental, 19951998: informe de vigilancia procedente de Enternet. Eurosurveillance 1999; 4:56.

3. Outbreaks of Salmonella Serotype enteritidis infection associated with eating shell eggs. United States, 1999-2001 MMWR 2003 51;1149-52. 
4. Hernández G, Soler P, Usera M, Tello O, Torres A. Vigilancia epidemiológica de brotes alimentarios relacionados con el consumo de huevos o derivados. España. 1998-2001. Bol Epidemiol Sem 2003; 11: 37-48.

5. Centro Nacional de Epidemiología. Protocolos de las enfermedades de declaración obligatoria. $2^{\mathrm{a}}$ Edición. Madrid: Ministerio de Sanidad y Consumo; 2001.

6. Hill AB. The environment and disease: Association or Causation? Proc R Soc Med 1965; 58: 295-300.

7. Bryan FL. Hazard Analysis Critical Control Point Evaluation. Geneve: WHO;1992.

8. D'Arpegio P, Romano A, Antonino F. An outbreak of Salmonella enteritidis infection associated with iced cake. Eurosurveillance 1999;4:24-6.

9. Dodhia H, Kearney J, Warburton F. A birthday party, home-made ice cream and an outbreak of Salmonella enteritidis phage type 6 infection. Commun Dis Public Health. 1998; 1:31-4.

10. Evans MR, Lane W, Ribeiro DC. Salmonella enteritidis PT6: another eggs-associated salmonellosis? Emerg Infect Dis 1998; 4:667-9.

11. Arnedo A, Bellido JB, Pac MR, Criado J, Usera MA, Mesanza I y col. Brotes epidémicos de Salmonelosis por consumo de huevos. Enferm Infecc Microbiol Clin 1998;16:408-12.
12. Boletín Oficial del Estado. Real Decreto 1254/1991, de 2 de agosto del Ministerio de Sanidad y consumo por el que se dictan normas para la preparación y conservación de la mayonesa de elaboración propia y otros alimentos de consumo inmediato en los que figure el huevo como ingrediente. BOE núm 185 de 02/08/1991.

13. Boletín Oficial del Estado. Real Decreto 202/2000, de 11 de febrero del Ministerio de Sanidad y consumo por el que se establecen las normas relativas a los manipuladores de alimentos. BOE núm $48 \mathrm{de}$ $25 / 02 / 2000$

14. Neira M. En qué dirección va la seguridad alimentaria. Rev Esp Salud Pública 2003; 77: 307-11.

15. Foster KR, Vechia P, Repacholi MH: Science and the precautionary principli. Science 2000;288:979-80.

16. Godoy P, Artigues A, Usera MA, González JL, Pablo N, Agustí M. Brote de Toxiinfección alimentaria por consumo de espaghetis a la carbonara causado por Salmonella enteritidis. Enf Inf Microb Clin 2000; 18(6): 257-61.

17. Chin J. El control de las enfermedades transmisibles 17. ${ }^{a}$ ed.- Washington DC: OPS, 2001:552-8.

18. Bellido JB, González F, Arnedo A, Galiano JV, Safont L, Herrero C y col. Brote de infección alimentaria por Salmonella enteritidis. Posible efecto protector de las bebidas alcohólicas. Med Clin (Barc) 1996; 107:641-4. 